\title{
Relative importance of the metabolic syndrome as a cardio- vascular risk factor in Sri Lankans
}

\author{
Upali Illangasekera ${ }^{1}$, N S B Dissanayake ${ }^{2}$ \\ Sri Lanka Journal of Diabetes, Endocrinology and Metabolism 2012; 2: 49-51
}

\begin{abstract}
The relative importance of the metabolic syndrome as a cardiovascular risk factor was determined by comparing its prevalence in a sample of 1018 subjects with cardiovascular disease with those without established cardiovascular disease. According to these data metabolic syndrome was not associated with higher risk of cardiovascular disease. However, there was positive correlation between individual cardiovascular risk factors (hypertension,smoking,dyslipidaemia and a family history of cardiovascular disease). Waist circumference on its own was only poorly correlated with cardiovascular disease compared to the other risk factors.
\end{abstract}

\section{Introduction}

Established risk factors for cardiovascular disease include hypertension, smoking, glucose intolerance, obesity and dyslipidaemia, age and family history. In an individual patient these risk factors could occur in isolation or more commonly together. In a certain category of patients they tend to cluster together more than by chance. This cluster is called the metabolic syndrome (MS) and is considered to increase future risk of cardiovascular disease (CVD) as well as diabetes (1). Rising incidence of diabetes and obesity is likely to increase the prevalence of the MS. This study was carried out with the objective of determining the prevalence of the MS in those with and without CVD and to evaluate the strength of the relationship between individual cardiovascular risk factors in those with CVD.

\section{Methodology}

The study was cross sectional in design and was carried out in Teaching Hospitals of Kandy and Peradeniya. All patients over the age of 30 years attending the Cardiology Clinic at Teaching Hospital, Kandy $(n=164)$ and the Diabetic Clinic $(n=667)$ at Teaching Hospital, Peradeniya over a period of one month were selected. One hundred and eighty subjects with no evidence of CVD too were selected from hospital visitors and bystanders. Informed consent was obtained from all patients and subjects. The International Diabetes Federation (IDF) definition was used to diagnose the
MS (2). Cardiovascular disease was diagnosed on clinical features such as the presence of symptoms and signs of ischaemic heart disease, being treated for such disease and investigations such as ECG, echocardiography, exercise ECG and coronary angiography. The proportion of patients with MS among those with and without CVD was determined. The relationship between individual cardiovascular risk factors and CVD was carried out by assessing partial correlation for each risk factor while controlling for others. The waist circumference which is considered to be a surrogate marker of the MS too was assessed as a cardiovascular risk factor (3).

\section{Results}

The total number of patients enrolled for the study was 831 consisting of 164 (110 males, 54 females) from the Cardiology Clinic, Teaching Hospital, Kandy, and 667 (437 males and 230 females) from the Diabetic Clinic at Teaching Hospital, Peradeniya. One hundred and eighty seven (98 males, 89 females) matched for age were selected as those without CVD from hospital visitors and bystanders. The association between the MS and the CVD is shown in Table 1. As the results indicate there was no statistically significant relationship between the MS and CVD. When the strength of the relationship between individual risk factors and CVD was assessed it was found that while diabetes was negatively correlated the least correlation was for the waist circumference compared with other cardiovascular risk factors (Table 2).

${ }^{1}$ Professor in Medicine, Faculty of Medicine, University of Peradeniya, Sri Lanka, ${ }^{2}$ Research Assistant in Medicine, Faculty of Medicine, University of Peradeniya, Sri Lanka. 
Table 1. Relationship between the metabolic syndrome and cardiovascular disease

\begin{tabular}{lccc}
\hline & CVD present & CVD absent & Total \\
\hline MS present & $157(59)$ & $432(57)$ & $582(57)$ \\
MS absent & $109(41)$ & $320(43)$ & $429(43)$ \\
Total & $266(100)$ & $752(100)$ & $1011(100)$ \\
\hline
\end{tabular}

Significance: p 0.655 (t-test)

MS: metabolic syndrome, CVD: cardiovascular disease

Percentages in parentheses

Table 2. Correlation between individual risk factors and cardiovascular disease

\begin{tabular}{lcc}
\hline Risk factor & Correlation coefficient & Significance \\
\hline Diabetes & -0.24 & $\mathrm{p}<0.001$ \\
Smoking & 0.28 & $\mathrm{p}<0.001$ \\
Hypertension & 0.18 & $\mathrm{p}<0.001$ \\
Dyslipidaemia & 0.29 & $\mathrm{p}<0.001$ \\
Family history & 0.77 & $\mathrm{p}<0.05$ \\
W.C. Male & 0.10 & $\mathrm{p}<0.05$ \\
W.C. Female & 0.04 & $\mathrm{~N} . S$ \\
\hline
\end{tabular}

W.C. - waist circumference, N.S. - not significant

\section{Discussion}

The results of this study indicate that the MS was not associated with CVD and had the least correlation with CVD compared to other risk factors such as hypertension, dyslipidaemia, smoking and the family history.

The MS is a simple entity that can be used to identify individuals who are free of CVD but who are at an increased risk of future CVD risk. Since the diagnosis of the MS is commonly based on anthropometric and clinical data it would be an ideal tool for the use in developing countries such as Sri Lanka. Early publications reported rather substantial associations between the MS and CVD (4). An atherogenic role for the MS has been proposed as there are abnormalities in insulin resistance, lipids, blood pressure, glucose tolerance, LDL particle size, HDL cholesterol, endothelial function, cell adhesion molecules, plasminogen activator inhibitor, fibrinogen and inflammatory markers including C-reactive protein. However more recent studies have shown that although the MS can predict the future development of diabetes and CVD it predicts less effectively than established predicting models such as Diabetes Risk Score and Framingham Risk Score (5). Furthermore it has been shown that clustering had no greater predictive value beyond the consideration of the individual risk factors (6).The results of the present study too supports the notion of only a limited role for the MS as a potential cardiovascular risk factor in Sri Lankans. Limitations of the study include the sampling bias and the presence of other possible confounding factors. We could not find why diabetes had a negative correlation with CVD. Possible explanation is that people who develop CVD modify their lifestyle and hence develop less diabetes. In the same way those with CVD in this retrospective study has less abdominal obesity due to better control of diet and having more exercise and have lower prevalence of metabolic syndrome. Therefore there is a need to test this hypothesis in a prospective study with a randomly selected bigger sample and especially looking at development of CVD in those with and without metabolic syndrome during the follow up. 


\section{Acknowledgements}

Dr. Rohini Tennakoon and Dr. Subashini Jayawickrema, Consultant Cardiologists, Teaching Hospital, Kandy for granting permission to enroll their patients for the study.

\section{References}

1. Reven G. Role of insulin resistance in human disease. Diabetes 1988; 37:1595-607.

2. Alberti KG, Zimmet P, Shaw J. The metabolic syndrome a new worldwide definition. Lancet 2005; 366: 1059-62.

3. Lee J, Ma S, Heng D, et al. Should central obesity be an optional or essential component of the metabolic syndrome. Diabetes Care 2007; 30(2): 343 - 7.

4. Lakka HM, Laaksonen DE, Lakka TA, et al. The metabolic syndrome and total and cardiovascular disease mortality in middle aged men. Journal of the American Medical Association 2002; 288: 2709-16.

5. Stern MP, Williams K, Gonzalez-Villalpando C, et al. Does the metabolic syndrome improve identification of individuals at risk of type 2 diabetes and/or cardiovascular disease? Diabetes Care 2004; 27(11): 2676 - 81.

6. Yarnell JW, Patterson CC, Bainton D, Sweetnam PM. Is metabolic syndrome a discrete entity in the general population? Heart 1998: 79: 248 - 52. 